\title{
An extension of Yager's implications
}

\author{
S. Massanet J. Torrens \\ Department of Mathematics and Computer Science \\ University of the Balearic Islands \\ Crta. Valldemossa km 7.5, E-07122 Palma de Mallorca, Spain
}

\begin{abstract}
In this paper, an extension of Yager's implications is proposed by means of generalizing the internal factor $x$, in the case of $f$-generated implications, or $\frac{1}{x}$, in the case of $g$-generated implications, to more general unary functions. The importance of this extension stems from the fact that both subclasses of Yager's implications are extended in a similar way and the resulting classes of fuzzy implications will be useful to characterize the recently introduced $(h, e)$-implications. In addition, the behaviour and additional properties of the generated implications are determined.
\end{abstract}

Keywords: Fuzzy implications, Yager's $f$ generated implications, Yager's $g$-generated implications, $(h, e)$-implications.

\section{Introduction}

Fuzzy implications have become one of the main operations in fuzzy logic. Their importance lies on the fact that they play a similar role to the classical implication in crisp logic. Since it is well-settled that the fuzzy concept has to generalize the corresponding crisp one, fuzzy implications restricted to $\{0,1\}^{2}$ must coincide with the classical implication. Nowadays, it is widely accepted that fuzzy implications are performed by means of a binary operation $I:[0,1]^{2} \rightarrow[0,1]$ satisfying, in addition of that boundary conditions, some monotonicity properties. These logical connectives have recently attracted the efforts of numerous researchers, as evidence the publication of the survey [1] and the books [2] and [3], entirely devoted to these operators.

In this field, one of the main topics is the study of the known families of fuzzy implications, the proposal of new ones and how they interrelate among them. The requirements of the definition of fuzzy implications are flexible enough to allow several classes of implications with different additional properties. The proposal of many classes of fuzzy implications is supported by the use of these operators to perform any fuzzy "if-then" rule in fuzzy systems and inference processes, through Modus Ponens and Modus Tollens (see [4]). This is also true in other fields where fuzzy implications play an important role, such as fuzzy mathematical morphology [5] or fuzzy DI-subsethood measures [6]. So, depending on the context, and on the proper rule and its behaviour, different implications with different properties can be adequate.

An extended approach to generate classes of fuzzy implications is the use of unary generators of aggregation functions. In this sense, Yager's $f$ and $g$ generated implications [7] are generated from additive generators of continuous Archimedean t-norms and t-conorms; $h$-generated implications [8], from multiplicative generators of Archimedean t-conorms and $h$ and $(h, e)$-implications [9], from additive generators of representable uninorms.

From these generated classes of fuzzy implications, Yager's implications have focused many efforts due to their applications in approximate reasoning since they accomplish strictness of a fuzzy implication and sharpness of inference, two concepts introduced by Yager [7]. These implications have been recently characterized [10] and several generalizations have been proposed. In particular, the extensions of $f$-generated implications vary from taking a more general aggregation function than the product t-norm as internal function [11, 12] to considering a more general increasing function $g:[0,1] \rightarrow[0,1]$ than the identity function as a internal factor [13]. With respect to the $g$-generated implications, an extension considering the minimum t-norm instead of the product as internal function was proposed in [14].

In this paper, we want to propose a new extension of Yager's implications. The novelty of this extension lies on the use of a unary continuous function with range in $[0,+\infty]$ in the definition of both $f$ and $g$-generated implications instead of the internal factors $x$ and $\frac{1}{x}$. In this way, a common generalization is proposed for both methods and the conditions of these functions under which the generated operators are in fact fuzzy implications are studied. These extended Yager's implications will fulfil, in some cases, some of the usual additional properties that a fuzzy implication can satisfy, like the left neutrality principle, the exchange principle or the law of importation. Furthermore, these new classes will be crucial to characterize the recently introduced $(h, e)$-implications, in a similar way how $h$-implications are characterized by Yager's implications (see [15]).

The communication is organized as follows. In the next section we recall the basic definitions and properties we will use in the subsequent sections. In Section 3, the new classes of fuzzy implications 
extending Yager's ones will be proposed and the conditions under which some additional properties are satisfied by these operations are determined. Then, in Section 4, the characterization of $(h, e)$ implications involving these two new classes of implications is proved. The paper ends with some conclusions and future work.

\section{Preliminaries}

To make this work self-contained, we recall here some of the concepts and results employed in the rest of the paper. First of all, the definition of fuzzy negation is given.

Definition 1 ([16, Definition 1.1]). A decreasing function $N:[0,1] \rightarrow[0,1]$ is called a fuzzy negation, if $N(0)=1, N(1)=0$. A fuzzy negation $N$ is called

(i) strict, if it is strictly decreasing and continuous.

(ii) strong, if it is an involution, i.e., $N(N(x))=x$ for all $x \in[0,1]$.

Next, we recall the definition of fuzzy implications.

Definition 2 ([16, Definition 1.15]). A binary operator $I:[0,1]^{2} \rightarrow[0,1]$ is said to be a fuzzy implication if it satisfies:

(I1) $I(x, z) \geq I(y, z)$ when $x \leq y$, for all $z \in$ $[0,1]$.

(I2) $I(x, y) \leq I(x, z)$ when $y \leq z$, for all $x \in$ $[0,1]$.

(I3) $I(0,0)=I(1,1)=1$ and $I(1,0)=0$.

Note that, from the definition, it follows that $I(0, x)=1$ and $I(x, 1)=1$ for all $x \in[0,1]$ whereas the symmetrical values $I(x, 0)$ and $I(1, x)$ are not derived from the definition. We will denote by $\mathcal{F} \mathcal{I}$ the set of all fuzzy implications. Special interesting properties for implication functions are:

- The exchange principle,

$$
I(x, I(y, z))=I(y, I(x, z)), \quad x, y, z \in[0,1] .
$$

- The law of importation with a t-norm $T$,

$$
I(T(x, y), z)=I(x, I(y, z)), \quad x, y, z \in[0,1] .
$$

- The left neutrality principle,

$$
I(1, y)=y, \quad y \in[0,1] .
$$

- The ordering property,

$$
x \leq y \Longleftrightarrow I(x, y)=1, \quad x, y \in[0,1] .
$$

- The identity principle,

$$
I(x, x)=1, \quad x \in[0,1] .
$$

- The contrapositive symmetry with respect to a fuzzy negation $N$,

$$
I(x, y)=I(N(y), N(x)), \quad x, y \in[0,1] .
$$

$(\mathbf{C P}(\mathbf{N}))$

Definition 3 ([2, Definition 1.4.15]). Let $I$ be a fuzzy implication. The function $N_{I}$ defined by $N_{I}(x)=I(x, 0)$ for all $x \in[0,1]$, is called the natural negation of $I$.

Next, we recall the definitions of Yager's $f$ and $g$-generated implications.

Definition 4 ([16]). Let $f:[0,1] \rightarrow[0, \infty]$ be a strictly decreasing and continuous function with $f(1)=0$. The function $I:[0,1]^{2} \rightarrow[0,1]$ defined by

$$
I(x, y)=f^{-1}(x \cdot f(y)), \quad x, y \in[0,1]
$$

with the understanding $0 \cdot \infty=0$, is called an $f$-generated implication. The function $f$ itself is called an $f$-generator of the I generated as above. In such a case, to emphasize the apparent relation we will write $I_{f}$ instead of $I$.

Definition 5 ([16]). Let $g:[0,1] \rightarrow[0, \infty]$ be a strictly increasing and continuous function with $g(0)=0$. The function $I:[0,1]^{2} \rightarrow[0,1]$ defined by

$$
I(x, y)=g^{(-1)}\left(\frac{1}{x} \cdot g(y)\right), \quad x, y \in[0,1]
$$

with the understanding $\frac{1}{0}=\infty$ and $\infty \cdot 0=\infty$, is called a $g$-generated implication, where the function $g^{(-1)}$ is the pseudo-inverse of $g$ given by

$$
g^{(-1)}(x)= \begin{cases}g^{-1}(x) & \text { if } x \in[0, g(1)] \\ 1 & \text { if } x \in[g(1), \infty]\end{cases}
$$

The function $g$ is called a g-generator of the function $I$. In this case, we will write $I_{g}$ instead of $I$ to emphasize the apparent relation.

\section{Extended Yager's implications}

If the definitions of Yager's implications are observed accurately, one can see that $x$ can be regarded as a particular case of a function $g(x)=x$ and $\frac{1}{x}$ can be understood as a particular case of another function $f(x)=\frac{1}{x}$. Taking into account the above observation in this section, we will propose an extension of Yager's implications involving the use of more general unary functions as internal factor.

\subsection{Extension of $f$-generated implications}

We will focus on an extension of $f$-generated implications generalizing $g(x)=x$ to a continuous and strictly increasing function $g:[0,1] \rightarrow[0,+\infty]$ with $g(0)=0$. 
Definition 6. Let $f:[0,1] \rightarrow[0, \infty]$ be a strictly decreasing and continuous function with $f(1)=0$ and $g:[0,1] \rightarrow[0,+\infty]$ be a continuous and strictly increasing function with $g(0)=0$. The function $I_{f, g}:[0,1]^{2} \rightarrow[0,1]$ defined by

$$
I_{f, g}(x, y)=f^{(-1)}(g(x) \cdot f(y)), \quad x, y \in[0,1]
$$

with the understanding $0 \cdot \infty=0$, is called an $(f, g)$ generated operation.

A first difference from Yager's $f$-generated implications is that in this case, the use of the pseudoinverse is mandatory since when $f(0)<+\infty$, $g(x) \cdot f(y)$ can be greater than $f(0)$. However, the pseudo-inverse can be avoided in the definition leading to

$I_{f, g}(x, y)=f^{-1}(\min \{g(x) \cdot f(y), f(0)\}), x, y \in[0,1]$.

Remark 1. This extension is different from the one proposed in [13]. Although a similar approach is considered, their $(f, g)$-implications are generated through strictly increasing functions $g:[0,1] \rightarrow$ $[0,1]$ with $g(0)=0$ and $g(1)=1$, not necessarily continuous. In our approach, we extend the range of the function $g$ to $[0,+\infty]$ and consequently, $g(1) \neq 1$ in general. Obviously, if $g(1)=1$ then both approaches coincide and in fact, the generated $(f, g)$ implications are $\varphi$-conjugated of $f$-generated implications with $f$-generator $f \circ g^{-1}$ and $\varphi=g$ (see Remark 2.1 in [13]).

$(f, g)$-generated implications are not always fuzzy implications in the sense of Definition 2.

Theorem 1. An $(f, g)$-operation $I_{f, g}$ is a fuzzy implication if, and only if, one of the following conditions hold:

1. $f(0)=+\infty$.

2. $f(0)<+\infty$ and $g(1) \geq 1$.

When an $(f, g)$-operation is in fact a fuzzy implication, we will use the term $(f, g)$-implication and $(f, g)$ will be called an admissible pair of generators.

Next, we show some examples of $(f, g)$ implications which are neither Yager's $f$-generated implications nor Xie and Liu's $(f, g)$-implications covering all the boundary possibilities. These implications are depicted in Figure 1.

Example 1. (i) If we take $f_{1}(x)=1-x$ and $g_{1}(x)=x^{2}+x$ then we obtain the following implication

$$
I_{f_{1}, g_{1}}(x, y)=\max \left\{1-x^{2}+x^{2} y-x+x y, 0\right\},
$$

for all $x, y \in[0,1]$.

(ii) If we consider $f_{1}$ and $g_{2}(x)=\frac{x}{1-x}$ then we get the following implication

$$
I_{f_{1}, g_{2}}(x, y)= \begin{cases}1 & \text { if } x=y=1, \\ \max \left\{\frac{1-2 x+x y}{1-x}, 0\right\} & \text { otherwise. }\end{cases}
$$

(iii) If we consider $f_{2}(x)=-\ln x$ and $g_{1}$, we get the next implication

$$
I_{f_{2}, g_{1}}(x, y)= \begin{cases}1 & \text { if } x=y=0 \\ y^{x^{2}+x} & \text { otherwise }\end{cases}
$$

(iv) If we consider $f_{2}$ and $g_{2}$, we get the next implication

$$
I_{f_{2}, g_{2}}(x, y)= \begin{cases}1 & \text { if } x=y \in\{0,1\} \\ y^{\frac{x}{1-x}} & \text { otherwise }\end{cases}
$$

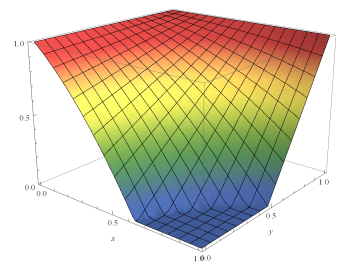

(a) $I_{f_{1}, g_{1}}$

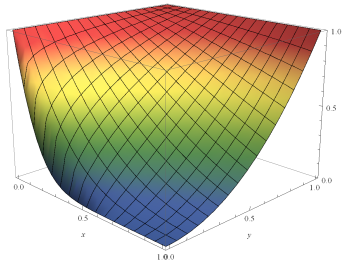

(c) $I_{f_{2}, g_{1}}$

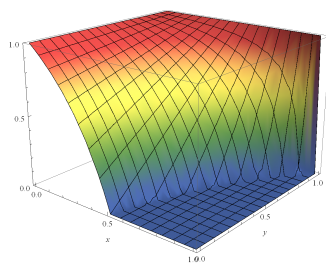

(b) $I_{f_{1}, g_{2}}$

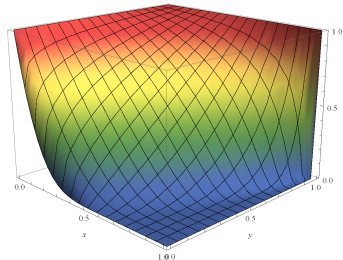

(d) $I_{f_{2}, g_{2}}$
Figure 1: Plots of some $(f, g)$-implications.

Note that in the previous examples, the $(f, g)$ implications have in some cases non-trivial zero region. The following result deals with this issue.

Proposition 1. Let $(f, g)$ be an admissible pair of generators. Then the following statements hold:

1. If $g(1)<f(0)=+\infty$, then $I_{f, g}(x, y)=0 \Leftrightarrow$ $y=0<x$.

2. If $g(1)=f(0)=+\infty$, then $I_{f, g}(x, y)=0 \Leftrightarrow$ $y<x=1$ or $y=0<x$.

3. If $f(0)<+\infty$, then $I_{f, g}(x, y)=0 \Leftrightarrow g(x) \geq 1$ and $y<f^{-1}\left(\frac{f(0)}{g(x)}\right)$.

On the other hand, we can also compute when these implications take the value 1 .

Proposition 2. Let $(f, g)$ be an admissible pair of generators. Then $I_{f, g}(x, y)=1$ if, and only if, $x=$ 0 or $y=1$.

The property obtained in the previous proposition is studied in detail in [17] where it is shown that such property is essential for the construction of strong equality indices.

Corollary 1. Let $(f, g)$ be an admissible pair of generators. Then the $(f, g)$-implication $I_{f, g}$ does not satisfy either (IP) or (OP).

Next, the following result establishes the condition under which $(f, g)$-implications satisfy (NP). 
Proposition 3. Let $(f, g)$ be an admissible pair of generators. Then $I_{f, g}$ satisfies (NP) if, and only if, $g(1)=1$.

Another interesting property of fuzzy implications is the continuity of these operators. The following result establishes that the possible points of discontinuity are restricted to $(0,0)$ or $(1,1)$.

Proposition 4. Let $(f, g)$ be an admissible pair of generators. Then the following properties hold:

1. If $g(1)=+\infty, I_{f, g}$ is not continuous at $(1,1)$.

2. If $f(0)=+\infty, I_{f, g}$ is not continuous at $(0,0)$.

3. $I_{f, g}$ is continuous if, and only if, $f(0), g(1)<$ $+\infty$.

Next we deal with the natural negation of these fuzzy implications.

Proposition 5. Let $(f, g)$ be an admissible pair of generators. Then the following properties hold:

1. If $f(0)=+\infty$, then the natural negation $N_{I_{f, g}}$ is the Gödel or least negation $N_{D_{1}}$.

2. If $f(0)<+\infty$, then the natural negation $N_{I_{f, g}}$ is given by

$$
N_{I_{f, g}}(x)= \begin{cases}f^{-1}(g(x) \cdot f(0)) & \text { if } g(x) \leq 1, \\ 0 & \text { if } g(x)>1 .\end{cases}
$$

3. If $f(0)<+\infty$ and $g(1)=1$, then the natural negation $N_{I_{f, g}}$ is a strict fuzzy negation.

Finally, the following results fully determine when the $(f, g)$-implications satisfy the exchange principle and the law of importation.

Proposition 6. Let $(f, g)$ be an admissible pair of generators. Then the following statements are equivalent:

1. $I_{f, g}$ satisfies $(E P)$.

2. $f(0)=+\infty$ or $(f(0)<+\infty$ and $g(1)=1)$.

Proposition 7. Let $(f, g)$ be an admissible pair of generators and $T$ a t-norm. Then the following statements are equivalent:

1. The couple of functions $I_{f, g}$ and $T$ satisfies (LI).

2. $g(1)=1$ and $T=\left(T_{P}\right)_{g}$, i.e., $T(x, y)=$ $g^{-1}(g(x) \cdot g(y))$ for all $x, y \in[0,1]$.

In [18], the equivalence between (EP) and (LI) was deeply studied. It was proved that (LI) is a strictly stronger property than (EP) and some counterexamples of fuzzy implications satisfying (EP) but not (LI) (or even the so-called weak law of importation) were pointed out. So, from the previous results, more examples are available since all $(f, g)$ implications with $f(0)=+\infty$ and $g(1) \neq 1$ satisfy (EP) but not (LI) with any t-norm $T$.
Example 2. Let us consider the $(f, g)$-implication $I_{f_{2}, g_{1}}$ presented in Example 1-(iii). It can be easily computed that $I_{f_{2}, g_{1}}$ satisfies (EP), but it does not satisfy (LI) since if we consider $x=1, y=\frac{1}{4}$ and $z=\frac{1}{2}$ we obtain on one hand,

$$
I_{f_{2}, g_{1}}(T(x, y), z)=I_{f_{2}, g_{1}}(y, z)=0.594604,
$$

and on the other hand,

$I_{f_{2}, g_{1}}\left(x, I_{f_{2}, g_{1}}(y, z)\right)=I_{f_{2}, g_{1}}\left(1, I_{f_{2}, g_{1}}(y, z)\right)=0.64842$.

\subsection{Extension of $g$-generated implications}

Now we will propose a similar extension of $g$ generated implications generalizing $f(x)=\frac{1}{x}$ to a continuous and strictly decreasing function $f$ : $[0,1] \rightarrow[0,+\infty]$ with $f(0)=+\infty$.

Definition 7. Let $g:[0,1] \rightarrow[0, \infty]$ be a strictly increasing and continuous function with $g(0)=0$ and $f:[0,1] \rightarrow[0,+\infty]$ be a continuous and strictly decreasing function with $f(0)=+\infty$. The function $I:[0,1]^{2} \rightarrow[0,1]$ defined by

$$
I_{g, f}(x, y)=g^{(-1)}(f(x) \cdot g(y)), \quad x, y \in[0,1]
$$

with the understanding $0 \cdot(+\infty)=+\infty$ and $\frac{1}{0}=$ $+\infty$, is called a $(g, f)$-generated operation.

Let us determine which $(g, f)$-generated operations are in fact fuzzy implications in the sense of Definition 2.

Theorem 2. $A(g, f)$-operation $I_{g, f}$ is a fuzzy implication if, and only if, one of the following conditions hold:

1. $g(1)=+\infty$.

2. $g(1)<+\infty$ and $f(1) \geq 1$.

When a $(g, f)$-operation is a fuzzy implication, we will use the term $(g, f)$-implication and $(g, f)$ will be called an admissible pair of generators.

In Figure 2 we show some examples of $(g, f)$ implications which are not Yager's $g$-generated implications covering all the boundary possibilities.

Example 3. (i) If we take $g_{1}(x)=-\ln (1-x)$ and $f_{1}(x)=\frac{1}{x^{2}}$ then we obtain the following implication

$$
I_{g_{1}, f_{1}}(x, y)= \begin{cases}1 & \text { if } x=0, y \in\{0,1\}, \\ 1-(1-y)^{\frac{1}{x^{2}}} & \text { otherwise. }\end{cases}
$$

(ii) If we consider $g_{1}$ and $f_{2}(x)=\frac{x^{2}+1}{x^{2}}$ then we get the following implication

$$
I_{g_{1}, f_{2}}(x, y)= \begin{cases}1 & \text { if } x=0, y \in\{0,1\}, \\ 1-(1-y)^{\frac{x^{2}+1}{x^{2}}} & \text { otherwise. }\end{cases}
$$

(iii) If we consider $g_{2}(x)=x$ and $f_{1}$, we get the next implication

$$
I_{g_{2}, f_{1}}(x, y)=\min \left\{\frac{y}{x^{2}}, 1\right\}
$$

for all $x, y \in[0,1]$. 
(iv) If we consider $g_{2}$ and $f_{2}$, we get the next implication

$$
I_{g_{2}, f_{2}}(x, y)=\min \left\{\frac{y \cdot\left(x^{2}+1\right)}{x^{2}}, 1\right\}
$$

for all $x, y \in[0,1]$.

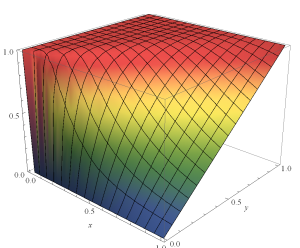

(a) $I_{g_{1}, f_{1}}$

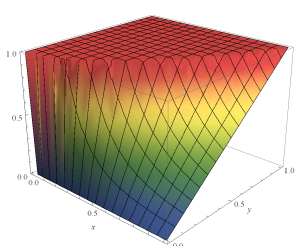

(c) $I_{g_{2}, f_{1}}$

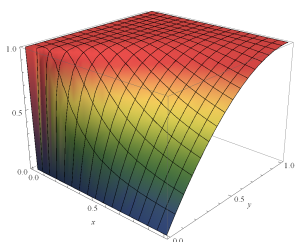

(b) $I_{g_{1}, f_{2}}$

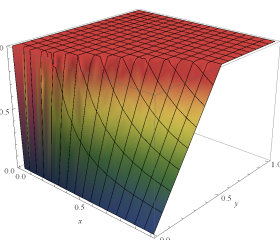

(d) $I_{g_{2}, f_{2}}$
Figure 2: Plots of some $(g, f)$-implications.

Let us find when these implications take value 1 . Note that in some cases the $(g, f)$-implications can have a non-trivial 1 region.

Proposition 8. Let $(g, f)$ be an admissible pair of generators. Then the following statements hold:

1. If $g(1)=+\infty$, then $I_{g, f}(x, y)=1 \Leftrightarrow x=0$ or $y=1$.

2. If $g(1)<+\infty$, then $I_{g, f}(x, y)=1 \Leftrightarrow y \geq$ $g^{-1}\left(\frac{g(1)}{f(x)}\right)$.

On the other hand, they do value 0 only when $x>0$ and $y=0$ as the following result shows.

Proposition 9. Let $(g, f)$ be an admissible pair of generators. Then $I_{g, f}(x, y)=0$ if, and only if, $x>$ 0 and $y=0$.

From the previous result, the following corollary is straightforward.

Corollary 2. Let $(g, f)$ be an admissible pair of generators. Then the natural negation $N_{I_{g, f}}$ is the Gödel or least fuzzy negation $N_{D_{1}}$.

Unlike $(f, g)$-implications, $(g, f)$-implications can satisfy (IP) and (OP) in some cases.

Proposition 10. Let $(g, f)$ be an admissible pair of generators. Then $I_{g, f}$ satisfies (IP) if, and only if, $g(1)<+\infty$ and $f(x) \geq \frac{g(1)}{g(x)}$ for all $x \in[0,1]$.

Proposition 11. Let $(g, f)$ be an admissible pair of generators. Then the following statements are equivalent:
1. I $I_{g, f}$ satisfies $(O P)$.

2. $g(1)<+\infty$ and there exists a constant $c \in$ $(0,+\infty)$ such that $f(x)=\frac{c}{g(x)}$.

3. $I_{g, f}(x, y)=f^{-1}\left(\max \left\{f(1), \frac{f(y)}{f(x)}\right\}\right)$

Next, we study the continuity of $(g, f)$ implications which are never continuous at point $(0,0)$ since the natural negation is $N_{D_{1}}$. In addition, they can be also not continuous at $(1,1)$ as the following result states.

Proposition 12. Let $(g, f)$ be an admissible pair of generators. Then the following properties hold:

1. $I_{g, f}$ is continuous everywhere except at $(0,0)$ if, and only if, $g(1)<+\infty$ or $(g(1)=+\infty$ and $f(1)>0)$.

2. $I_{g, f}$ is continuous everywhere except at $(0,0)$ and $(1,1)$ if, and only if, $g(1)=+\infty$ and $f(1)=0$.

Finally, let us study (EP) and (LI) for this class of fuzzy implications.

Proposition 13. Let $(g, f)$ be an admissible pair of generators. Then $I_{g, f}$ always satisfy (EP).

Proposition 14. Let $(g, f)$ be an admissible pair of generators and $T$ a t-norm. Then the following statements are equivalent:

1. The couple of functions $I_{g, f}$ and $T$ satisfies $(L I)$.

2. $f(1)=1$ and $T(x, y)=f^{-1}(f(x) \cdot f(y))$ for all $x, y \in[0,1]$.

Again novel examples of fuzzy implications satisfying (EP) but not (LI) with any t-norm $T$ arise. In particular, any $(g, f)$-implication with $f(1)>1$ satisfies (EP) but not (LI).

Example 4. Let us consider the $(g, f)$-implication $I_{g_{2}, f_{2}}$ presented in Example 3-(iv). It can be easily computed that $I_{g_{2}, f_{2}}$ satisfies (EP), but it does not satisfy (LI) since if we consider $x=1, y=\frac{3}{4}$ and $z=\frac{1}{8}$ we obtain on one hand,

$$
I_{g_{2}, f_{2}}(T(x, y), z)=I_{g_{1}, f_{2}}(y, z)=0.3472,
$$

and on the other hand,

$I_{g_{2}, f_{2}}\left(x, I_{g_{2}, f_{2}}(y, z)\right)=I_{g_{2}, f_{2}}\left(1, I_{g_{2}, f_{2}}(y, z)\right)=0.6944$.

\section{Characterization of $(h, e)$-implications}

The $(f, g)$ and $(g, f)$-generated implications presented in the previous section are going to be decisive in order to characterize the class of $(h, e)$ implications, presented recently in [9]. Let us recall the definitions of $h$ and $(h, e)$-implications.

Definition 8 ([9, Definition 7]). Fix an e $\in(0,1)$ and let $h:[0,1] \rightarrow[-\infty, \infty]$ be a strictly increasing and continuous function with $h(0)=-\infty, h(e)=0$ 
and $h(1)=+\infty$. The function $I:[0,1]^{2} \rightarrow[0,1]$ defined by

$I^{h}(x, y)= \begin{cases}1 & \text { if } x=0 \\ h^{-1}(x \cdot h(y)) & \text { if } x>0 \text { and } y \leq e \\ h^{-1}\left(\frac{1}{x} \cdot h(y)\right) & \text { if } x>0 \text { and } y>e\end{cases}$

is called an h-implication. The function $h$ itself is called an h-generator (with respect to e) of the implication function I defined as above.

Definition 9 ([9, Definition 8]). Let $h:[0,1] \rightarrow$ $[-\infty, \infty]$ be a strictly increasing and continuous function with $h(0)=-\infty, h(e)=0$ for an $e \in(0,1)$ and $h(1)=+\infty$. The function $I:[0,1]^{2} \rightarrow[0,1]$ defined by

$I^{h, e}(x, y)= \begin{cases}1 & \text { if } x=0 \\ h^{-1}\left(\frac{x}{e} \cdot h(y)\right) & \text { if } x>0 \text { and } y \leq e \\ h^{-1}\left(\frac{e}{x} \cdot h(y)\right) & \text { if } x>0 \text { and } y>e\end{cases}$

is called an $(h, e)$-implication. The function $h$ itself is called an h-generator of the I defined as above.

The $(h, e)$-implications were proposed with the aim of modifying $h$-implications in order to satisfy the left neutrality principle for implications derived from uninorms $\left(\mathrm{NP}_{e}\right)$, that is, $I(e, y)=y$ for all $y \in[0,1]$. Both $h$ and $(h, e)$-implications are generated by means of the additive generator $h$ of a representable uninorm.

Recently, a novel method to generate fuzzy implications from two given ones, called the threshold generation method, was proposed in [15]. It is based on an adequate scaling on the second variable of the initial implications.

Theorem 3 ([15, Theorem 3]). Let $I_{1}, I_{2}$ be two implications and $e \in(0,1)$. Then the binary function $I_{I_{1}-I_{2}}:[0,1]^{2} \rightarrow[0,1]$, called the e-threshold generated implication from $I_{1}$ and $I_{2}$, defined as $I_{I_{1}-I_{2}}(x, y)$

$= \begin{cases}1 & \text { if } x=0, \\ e \cdot I_{1}\left(x, \frac{y}{e}\right) & \text { if } x>0 \text { and } y \leq e, \\ e+(1-e) \cdot I_{2}\left(x, \frac{y-e}{1-e}\right) & \text { if } x>0 \text { and } y>e,\end{cases}$

is a fuzzy implication.

In such a paper, $h$-implications were characterized in terms of some Yager's $f$ and $g$-generated implications as particular cases of threshold generated implications.

Theorem 4 ([15, Theorem 2, Remark 3]). Let $I:[0,1]^{2} \rightarrow[0,1]$ be a binary function and $e \in(0,1)$. Then $I$ is an h-implication with respect to $e$, if and only if, there exist $f$ and $g$-generated implications with $f(0)=g(1)=\infty, I_{f}$ and $I_{g}$ respectively, such that $I=I_{I_{f}-I_{g}}$.
Moreover, in this case generators $h, f$ and $g$ are related in the following way:

$$
\begin{aligned}
& f(x)=-h(e \cdot x) \quad \text { for all } x \in[0,1], \\
& g(x)=h(e+(1-e) \cdot x) \quad \text { for all } x \in[0,1], \\
& h(x)= \begin{cases}-f\left(\frac{x}{e}\right) & \text { if } x \leq e, \\
g\left(\frac{x-e}{1-e}\right) & \text { if } x>e .\end{cases}
\end{aligned}
$$

On the other hand, until now, although $(h, e)$ implications are also particular cases of threshold generated implications, since they also satisfy ${ }^{1}$ $I^{h, e}(x, e)=e$ for all $x>0$, they have not been characterized yet. However, after the introduction of $(f, g)$ and $(g, f)$-implications in the previous section, they can be characterized in a similar way to $h$-implications.

Theorem 5. Let $I:[0,1]^{2} \rightarrow[0,1]$ be a binary function and $e \in(0,1)$. Then $I$ is an $(h, e)$ implication with respect to $e$ if, and only if, there exist an $f$-generator with $f(0)=+\infty$ and a $g$ generator with $g(1)=+\infty$ such that $I$ is given by $I(x, y)=$

$= \begin{cases}1 & \text { if } x=0, \\ e \cdot f^{-1}\left(\frac{x}{e} \cdot f\left(\frac{y}{e}\right)\right) & \text { if } x>0, y \leq e, \\ e+(1-e) \cdot g^{-1}\left(\frac{e}{x} \cdot g\left(\frac{y-e}{1-e}\right)\right) & \text { if } x>0, y>e .\end{cases}$

Moreover, in this case generators $h, f$ and $g$ are related in the following way:

$$
\begin{aligned}
& f(x)=-h(e \cdot x) \quad \text { for all } x \in[0,1], \\
& g(x)=h(e+(1-e) \cdot x) \quad \text { for all } x \in[0,1], \\
& h(x)= \begin{cases}-f\left(\frac{x}{e}\right) & \text { if } x \leq e, \\
g\left(\frac{x-e}{1-e}\right) & \text { if } x>e .\end{cases}
\end{aligned}
$$

Remark 2. Note that under the same conditions of the previous theorem, if we consider the admissible pair of generators $\left(f, g_{1}\right)$ such that $g_{1}(x)=\frac{x}{e}$ and the admissible pair of generators $\left(g, f_{1}\right)$ such that $f_{1}(x)=\frac{e}{x}$, then $I=I_{I_{f, g_{1}}-I_{g, f_{1}}}$. That is, an $(h, e)$-implication is the e-threshold generated implication of some adequate $(f, g)$ and $(g, f)$-generated implications.

This characterization allows us to fully determine the initial $(f, g)$ and $(g, f)$-implications from which the $(h, e)$-implication is obtained through the threshold generation method.

Example 5. If we take the h-generator (with respect to $\left.e=\frac{1}{2}\right) h_{1}(x)=\ln \left(\frac{x}{1-x}\right)$, then we obtain the following $(h, e)$-implication

$$
I^{h_{1}}(x, y)= \begin{cases}1 & \text { if } x=0, \\ \frac{y^{2 x}}{(1-y)^{2 x}+y^{2 x}} & \text { if } x>0 \text { and } y \leq \frac{1}{2}, \\ \frac{y^{\frac{1}{2 x}}}{(1-y)^{\frac{1}{2 x}}+y^{\frac{1}{2 x}}} & \text { if } x>0 \text { and } y>\frac{1}{2} .\end{cases}
$$

\footnotetext{
${ }^{1}$ Threshold generated implications were characterized in [15] as those that satisfy $I(x, e)=e$ for all $x>0$.
} 
By the previous theorem, this implication is constructed from the $(f, g)$-generated implication $I_{f, g_{1}}$ with $f(x)=-\ln \left(\frac{x}{2-x}\right)$ and $g_{1}(x)=2 x$, and from the $(g, f)$-generated implication $I_{g, f_{1}}$ with $g(x)=\ln \left(\frac{1+x}{1-x}\right)$ and $f_{1}(x)=\frac{1}{2 x}$ (see Figure 3). That is, $I^{h_{1}, e}$ is constructed from

$$
\begin{aligned}
I_{f, g_{1}}(x, y) & =\frac{2 y^{2 x}}{(2-y)^{2 x}+y^{2 x}}, \\
I_{g, f_{1}}(x, y) & =\frac{(1+y)^{\frac{1}{2 x}}-(1-y)^{\frac{1}{2 x}}}{(1+y)^{\frac{1}{2 x}}+(1-y)^{\frac{1}{2 x}}},
\end{aligned}
$$

using the threshold generation method.

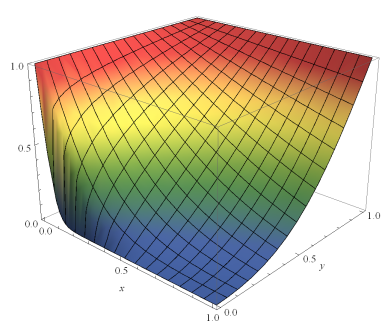

(a) $I_{f, g_{1}}$

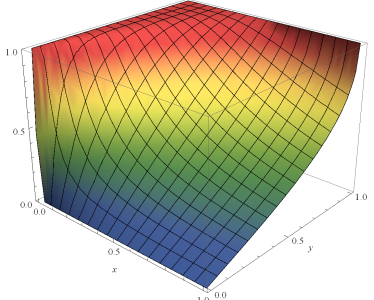

(b) $I_{g, f_{1}}$

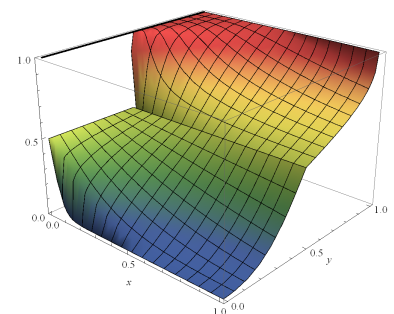

(c) $I^{h_{1}}$

Figure 3: Plot of the $(h, e)$-implication $I^{h_{1}, e}$ and its generating implications, $I_{f, g_{1}}$ and $I_{g, f_{1}}$.

\section{Conclusions and Future work}

In this paper, we have proposed an extension of Yager's $f$ and $g$-generated implications based on considering a more general internal factor than the usual $g(x)=x$ and $f(x)=\frac{1}{x}$ in $f$ and $g$ generated implications respectively. The generated new classes of implications, called $(f, g)$ and $(g, f)$ generated implications, have been deeply studied showing the additional properties they satisfy, including (EP), (OP) and (LI). In particular, in some cases we generate fuzzy implications satisfying (EP) but not (LI). Furthermore, these new classes have led to the characterization of $(h, e)$-implications showing that $(h, e)$-implications are just threshold generated implications from $(f, g)$ and $(g, f)$ implications.

As a future work, we want to study some additional properties on $(f, g)$ and $(g, f)$-implications such as the distributivity properties or the contrapositive symmetry. Finally, the intersections of these classes with the already known classes is worthy to study.

\section{Acknowledgement}

This paper has been supported by the Spanish Grants MTM2009-10320 with Feder support.

\section{References}

[1] M. Mas, M. Monserrat, J. Torrens, and E. Trillas. A survey on fuzzy implication functions. IEEE Transactions on Fuzzy Systems, 15(6):1107-1121, 2007.

[2] M. Baczyński and B. Jayaram. Fuzzy Implications, volume 231 of Studies in Fuzziness and Soft Computing. Springer, Berlin Heidelberg, 2008.

[3] M. Baczyński, G. Beliakov, H. Bustince, and A. Pradera. Advances in Fuzzy Implication Functions, volume 300 of Studies in Fuzziness and Soft Computing Series. Springer-Verlag GmbH, 2013.

[4] E.E. Kerre, C. Huang, and D. Ruan. Fuzzy Set Theory and Approximate Reasoning. Wu Han University Press, Wu Chang, 2004.

[5] M. Nachtegael and E. E. Kerre. Classical and fuzzy approaches towards mathematical morphology. In Etienne E. Kerre and Mike Nachtegael, editors, Fuzzy techniques in image processing, number 52 in Studies in Fuzziness and Soft Computing, chapter 1, pages 3-57. Physica-Verlag, New York, 2000.

[6] D. Sinha and E. Dougherty. Fuzzification of set inclusion: Theory and applications. Fuzzy Sets and Systems, 55:15-42, 1991.

[7] R.R. Yager. On some new classes of implication operators and their role in approximate reasoning. Information Sciences, 167:193-216, 2004.

[8] J. Balasubramaniam. Contrapositive symmetrisation of fuzzy implications-revisited. Fuzzy Sets and Systems, 157(17):2291 - 2310, 2006.

[9] S. Massanet and J. Torrens. On a new class of fuzzy implications: h-implications and generalizations. Information Sciences, 181(11):2111 2127, 2011.

[10] S. Massanet and J. Torrens. On the characterization of Yager's implications. Information Sciences, 201:1-18, 2012.

[11] S. Massanet and J. Torrens. On a generalization of Yager's implications. In S. Greco, B. Bouchon-Meunier, G. Coletti, M. Fedrizzi, B. Matarazzo, and R. R. Yager, editors, Advances on Computational Intelligence Proceedings of 14 th International Conference on Information Processing and Management of Uncertainty in Knowledge-Based Systems, IPMU 2012, Part II, volume 298 of Communi- 
cations in Computer and Information Science, pages 315-324. Springer, 2012.

[12] D. Hliněná, M. Kalina, and P. Král'. Generated implications revisited. In S. Greco, B. Bouchon-Meunier, G. Coletti, M. Fedrizzi, B. Matarazzo, and R.R. Yager, editors, $A d$ vances in Computational Intelligence, volume 298 of Communications in Computer and Information Science, pages 345-354. Springer Berlin Heidelberg, 2012.

[13] A. Xie and H. Liu. A generalization of Yager's f-generated implications. International Journal of Approximate Reasoning, 54(1):35 - 46, 2013.

[14] H.-W. Liu. On a new class of implications: (g, min)-implications and several classical tautologies. International Journal of Uncertainty, Fuzziness and Knowledge-Based Systems, 20(1):1-20, 2012.

[15] S. Massanet and J. Torrens. Threshold generation method of construction of a new implication from two given ones. Fuzzy Sets and Systems, 205:50-75, 2012.

[16] J. C. Fodor and M. Roubens. Fuzzy Preference Modelling and Multicriteria Decision Support. Kluwer Academic Publishers, Dordrecht, 1994.

[17] H. Bustince, J. Fernandez, J. Sanz, M. Baczyński, and R. Mesiar. Construction of strong equality index from implication operators. Fuzzy Sets and Systems, 211:15-33, 2013.

[18] S. Massanet and J. Torrens. The law of importation versus the exchange principle on fuzzy implications. Fuzzy Sets and Systems, 168(1):47 - 69, 2011. 